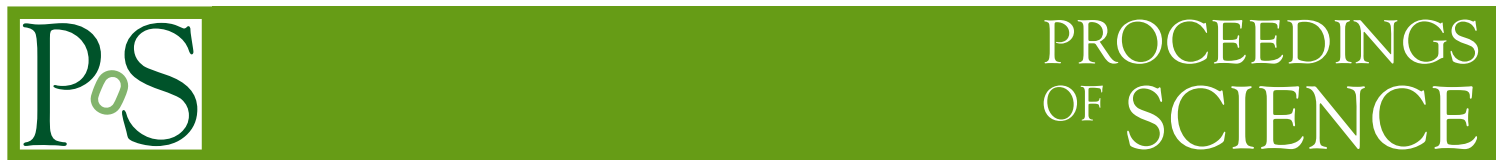

\title{
Single Top Quark Production at the Tevatron
}

\author{
Yvonne Peters* ${ }^{* \dagger}$ \\ University of Göttingen and DESY, Germany \\ E-mail: reinhild.peters@cern.ch
}

While the heaviest known elementary particle, the top quark, has been discovered in 1995 by the $\mathrm{CDF}$ and D0 collaborations in $t \bar{t}$ events, it took 14 more years until the observation of single top quark production. In this article, we discuss recent studies of single top quark production by the CDF and D0 collaborations at the Tevatron. In particular, we present the measurement of single top quark $s$ - and $t$-channel production combined, the first observation of $t$-channel production, the simultaneous measurement of $s$ - and $t$-channel production cross sections as well as the extraction of the CMK matrix element $\left|V_{t b}\right|$.

36th International Conference on High Energy Physics,

July 4-11, 2012

Melbourne, Australia

\footnotetext{
*Speaker.

† On behalf of the CDF and D0 collaborations.
} 


\section{Introduction}

Single top quark production via the electroweak interaction occurs via the $s$-channel, $t$-channel and $W t$-channel. The latter has a negligible cross section at the Tevatron. While the discovery of top quarks by the CDF and D0 collaborations happened in 1995 [1, 2] in $t \bar{t}$ production, it took additional 14 years to observe single top quark production. Both the CDF and D0 collaborations reported single top quark observation in Spring 2009 [3, 4]. For the single top quark observation, the $s+t$-channel cross section $\left(\sigma_{s+t}\right)$ was measured by CDF and D0 using up to $3.2 \mathrm{fb}^{-1}$ and $2.3 \mathrm{fb}^{-1}$ of data, respectively. While the cross section of the $s+t$-channel is only about a factor of two smaller than $t \bar{t}$ production, its signature is very similar to $W+$ jets events, making single top a challenging process to measure.

In the following, we present updated measurements of the $s+t$ channel cross section by CDF and D0, as well as the individual meaurement of $t$-channel cross section, the simultaneous extraction of $s$ - and $t$-channel cross sections as well as the extraction of the Cabibbo-Kobayashi-Maskawa (CKM) matrix element $\left|V_{t b}\right|$ from single top.

\section{Selection and $(s+t)$-channel Measurement}

At the Tevatron $p \bar{p}$ collider with a center of mass energy of $1.96 \mathrm{TeV}$, the cross section of $s$-channel and $t$-channel single top quark production is $1.04 \mathrm{pb}$ and $2.26 \mathrm{pb}$ in the standard model (SM), respectively [5]. Figure 1 shows the leading order Feynman diagrams for the $s$-channel (a) and $t$-channel (b) single top quark production. The signature of the signal is very similar to that of $W$ boson production in association with jets, presenting a challenge for the single top quark cross section measurements. Other background contributions to single top quark measurements result from $t \bar{t}$ production and instrumental background from jets faking isolated leptons, and smaller contributions from diboson $(W W, W Z$ and $Z Z$ ) and $Z+$ jets production. The selection of single top quark events focuses on events where the $W$ boson from the top quark decays into a charged electron or muon and the corresponding neutrino. Therefore, the requirements are a charged, isolated lepton (electron or muon) with high transverse momentum $\left(p_{T}\right)$, two, three or four jets, of which the leading jet has to have high $p_{T}$, and large missing transverse energy, a characteristic of events with undetected neutrinos. Additionally, cuts on angular distributions are applied in order to reduce the instrumental background. Such a cut is for example applied on the angular difference between the transverse lepton direction and the missing transverse energy at D0. In $t$-channel production, one of the jets can have high $\eta$ and low $p_{T}$, which is challenging to model.

After this pre-selection, the $W+$ jets and instrumental backgrounds are estimated using a datadriven approach. To further increase the purity of the sample, the application of $b$-jet identification is necessary. A neural-net based $b$-tagger is used at D0 [6] which is based on lifetime and displaced track information. At CDF, a secondary vertex $b$-tagger is used. We require one or two $b$-jets in the event. In total, up to six channels are considered: The D0 collaboration considers events with two, three and four jets, split into events with one or two identified $b$-jets, while in the recent analysis by the CDF collaboration events with exactly two or three jets, split into events with one or two identified $b$-jets are considered. 
(a)

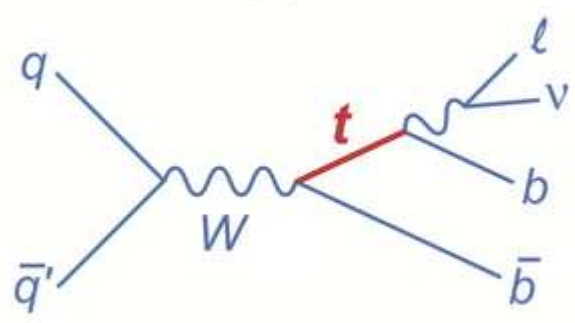

(b)

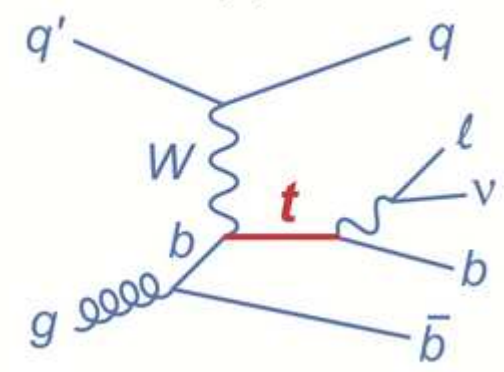

Figure 1: Tree-level Feynman diagrams for $s$-channel (a) and $t$-channel (b) single top quark production.

In order to further enrich the data sample in single top quark events, multivariate analysis (MVA) techniques are necessary, that separate signal from background. In the recent single top measurement by CDF, using $7.5 \mathrm{fb}^{-1}$ of Run II data, a neural network is trained on single top quark signal. At D0, using $5.4 \mathrm{fb}^{-1}$ of data, three different multivariate analysis techniques are applied separately on the data, and these are used as input to a super-discriminant to increase the sensitivity of the analysis. In particular, a boosted decision tree (BDT), a bayesian neural network (BNN) as well as a NEAT (neuro evolution of augmented topologies) discriminant are applied on the data. For the reduction of the correlation between the methods, the BNN is trained using the four-vectors of all final state particles, the two-vector of the missing transverse energy and a few additional variables that include lepton charge and $b$-tagging information, while the BDT approach uses a variety of complex variables as input to the BDT. The super-discriminant is build using a BNN. Depending on the final channel of interest, the training of the MVAs is performed by training either on $s+t$ channel as signal, by training on $t$-channel as signal and treating $s$-channel as background in the training procedure, or by training on $s$-channel as signal and treating $t$-channel as background during the training. The CDF collaboration performs the training differently in the different data samples: for the various jet and $b$-tag bins the largest expected contribution of $s$ - and $t$-channel is estimated and the training performed on this particular channel in the considered jet and $b$-tag bins.

The final extraction of the cross section from the discriminant is performed using a Bayesian method, where systematic uncertainties are incorporated as Gaussian priors and are integrated over. The measured $s+t$-channel cross section is $\sigma_{s+t}=3.43_{-0.74}^{+0.73} \mathrm{pb}$ at D0, where the training is performed on the $s+t$-channel as signal, and $\sigma_{s+t}=3.04_{-0.53}^{+0.57}$ at $\mathrm{CDF}[7,8]$. Both measurements are using a top quark mass of $172.5 \mathrm{GeV}$ in the Monte Carlo simulation of the signal. Figure 2 shows the comparison of prediction and data of the discriminant outputs for the D0 (left and middle) and CDF (right) measurements. The main systematic uncertainties arise from the uncertainty on the integrated luminosity, from uncertainties on the jet energy scale and resolution, and on the identification of $b$-jets. Both measurements are in agreement with each other and with the SM prediction. 

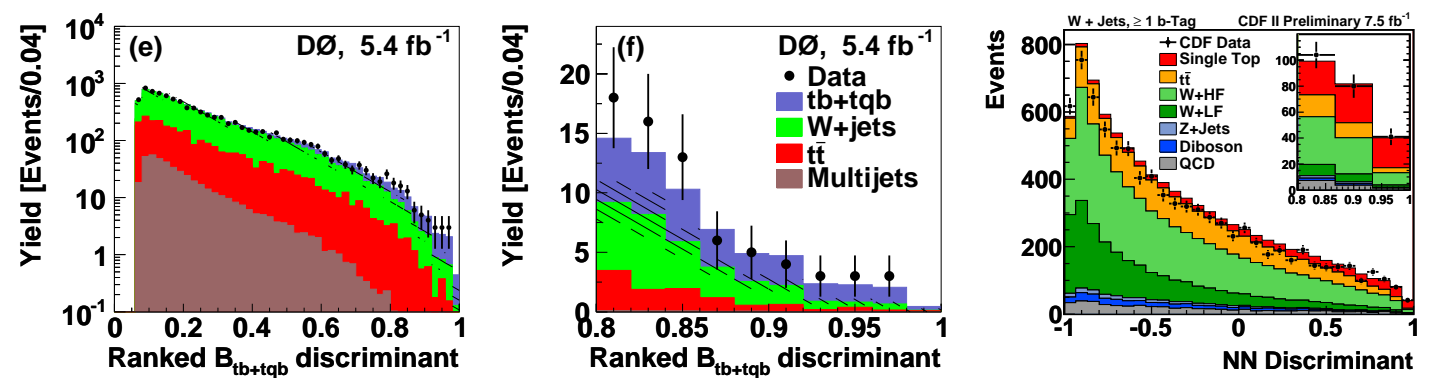

Figure 2: Comparison for Data and prediction of the discriminant outputs for the combined channel at D0 (left, middle) [7] and CDF (right) [8]. The discriminants for D0 are trained on the $s+t$-channel single top signal, and are ranked according to the signal over background ratio in the bins. The left plot shows the full distribution, and the middle plot the zoomed region of high ranked discriminant output.

\section{3. $t$-channel Observation and 2D Measurements}

Signatures of models for physics beyond the SM could look similar to $s$-channel or $t$-channel processes. Depending on the choice of model, either $s$ - or $t$-channel optimized selections would be more sensitive to new physics. Therefore, the simultaneous measurement of $s$-versus $t$-channel production cross sections is a good way to probe for new physics. Both, CDF and D0 collaborations, performed the $2 \mathrm{D}$ cross section measurement, extracting $s$ - and $t$-channel simultaneously, without assuming the SM ratio between both channels as is done for the $s+t$-channel extraction. While at D0, the training was performed with $t$-channel as signal, CDF performs the training on $s$ - and $t$-channel depending on the number of jets and $b$-tags in the event. Using $5.4 \mathrm{fb}^{-1}$ of data, D0 measures $\sigma_{s}=0.98 \pm 0.63 \mathrm{pb}$ and $\sigma_{t}=2.90 \pm 0.59 \mathrm{pb}$ [7]. The CDF collaboration extracts $\sigma_{s}=1.81_{-0.58}^{+0.63} \mathrm{pb}$ and $\sigma_{t}=1.49_{-0.42}^{+0.47} \mathrm{pb}$ using $7.5 \mathrm{fb}^{-1}$ of data [8] . Both results are compatible with the SM prediction. Due to the different training, CDF is more sensitive to $s$-channel single top production than D0. In Fig. 3 the SM cross sections, the measured cross sections and the contours of equal probability are shown for the $s$ - versus $t$-channel single top quark production cross section measurement from D0 (left) and CDF (right).

By integrating over the $s$-channel, the D0 collaboration also extracts the $t$-channel cross section from the 2D measurement, resulting in $\sigma_{t}=2.90 \pm 0.59$ [9]. This result deviates with more than 5.5 standard deviations from zero, and is the first observation of the $t$-channel single top cross section. The result is in good agreement with the SM prediction.

\section{Measurement of $\left|V_{t b}\right|$}

One interesting application of the single top quark analyses is the direct extraction of the CKM matrix element $\left|V_{t b}\right|$, without any assumptions about the number of fermion generations. The square of $\left|V_{t b}\right|$ is proportional to the single top cross section $\sigma_{s+t}$. Using the results for the $s+t$-channel single top cross section extracted from $5.4 \mathrm{fb}^{-1}$ of D0 and $7.5 \mathrm{fb}^{-1}$ of CDF data, we extract lower limits of $\left|V_{t b}\right|>0.79$ at $\mathrm{D} 0$ and $\left|V_{t b}\right|>0.78$ at CDF at the $95 \%$ confidence level using a Bayesian method. In Figure 4 the posterior curves for $\left|V_{t b}\right|^{2}$ are shown for the measurement from the D0 (left) and CDF (right) collaborations. 

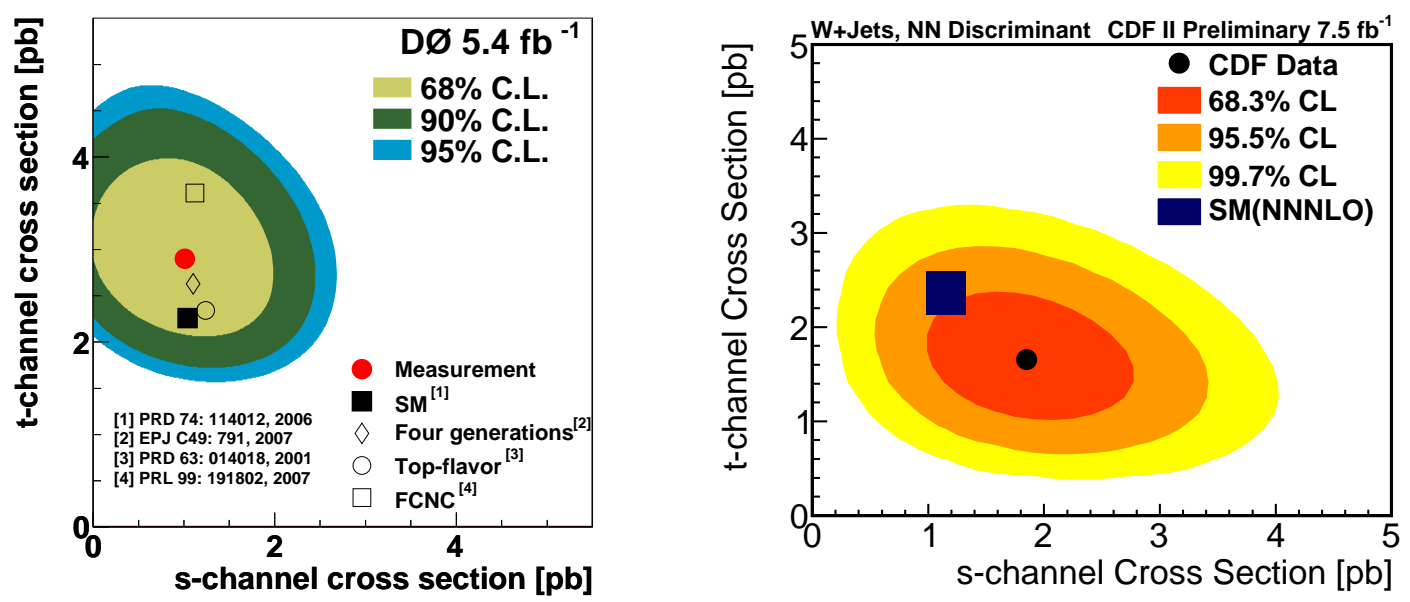

Figure 3: Posterior probability density for $s$ - versus $t$-channel single top quark production cross sections. Contours of equal probabilty are shown, plus the measured and predicted cross sections, for D0 (left) [9] and CDF (right) [8]. Note the difference in choice of contours between the two plots.

While the described extraction of $\left|V_{t b}\right|$ is not using any assumption about the number of quark generations, it does assume $\left|V_{t s}\right|^{2},\left|V_{t d}\right|^{2}<<\left|V_{t b}\right|^{2}$. An alternative way to extract $\left|V_{t b}\right|$ is by using the result from the indirect determination of the top quark width $[10,11] .\left|V_{t b}\right|^{2}$ is proportinal to the top width, the latter of which is determined by combining the measurement of the ratio of branching fractions $R=B(t \rightarrow W b) / B(t \rightarrow W q)$, with $q$ any down-type quark, in $t \bar{t}$ and the $t$-channel single top cross section. This $\left|V_{t b}\right|^{2}$ extraction does not assume $\left|V_{t s}\right|^{2},\left|V_{t d}\right|^{2}<<\left|V_{t b}\right|^{2}$, and also does not assume the SM ratio between the $s$ - and $t$-channel cross sections. Using the result from the indirect top width determination, we extract $\left|V_{t b}\right|>0.81$.

\section{Conclusion and Outlook}

The measurement of single top quark production at the Tevatron is very challenging due to the small production cross section and the similarity of the single top signal and the main background from $W+$ jets production. Using a variety of multivariate analysis techniques, both CDF and D0 collaborations measured single top production cross sections for $t$-channel and $s+t$-channels. The simultaneous extraction of the $s$ - and $t$-channel cross section yields a sensitive probe for new physics. Furthermore, the CKM matrix element $\left|V_{t b}\right|$ has been extracted. The full Tevatron dataset has still to be explored, with the main goal to get evidence or observation for the s-channel single top quark production.

\section{Acknowledgements}

I thank my collaborators from $\mathrm{CDF}$ and $\mathrm{D} 0$ for their help in preparing the presentation and this article. I also thank the staffs at Fermilab and collaborating institutions, and acknowledge the support from the Helmholtz association. 

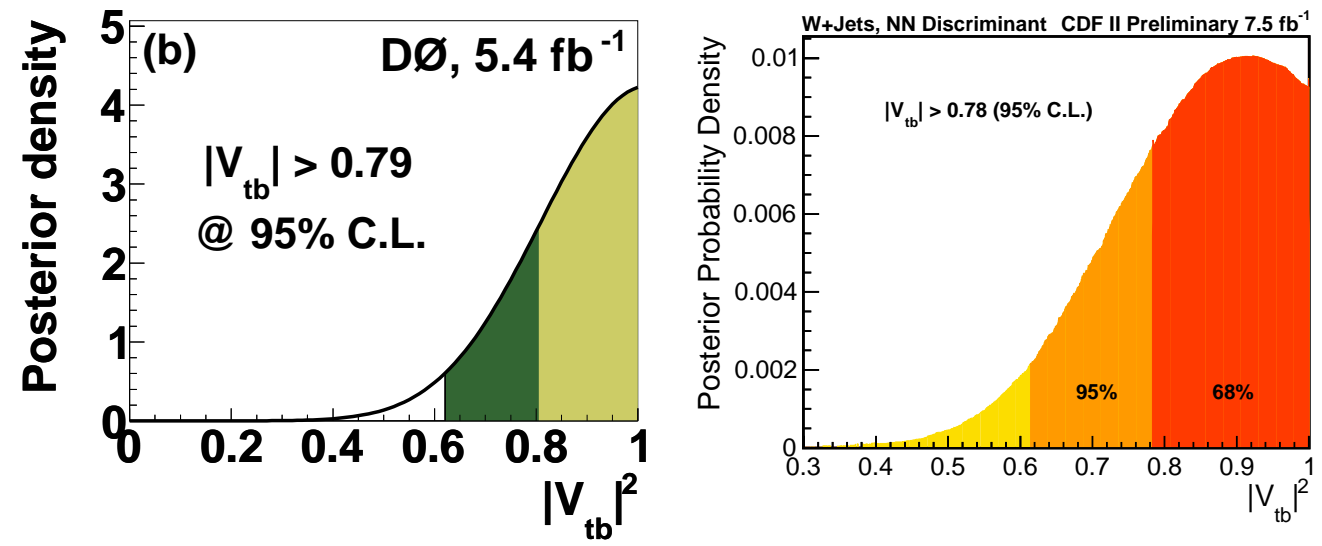

Figure 4: The posterior density functions for $\left|V_{t b}\right|^{2}$, for the measurement by the D0 (left) [7] and CDF (right) [8] collaborations.

\section{References}

[1] F. Abe et al. [CDF Collaboration], Phys. Rev. Lett. 74, 2626 (1995).

[2] S. Abachi et al. [D0 Collaboration], Phys. Rev. Lett. 74, 2632 (1995).

[3] T. Aaltonen et al. [CDF Collaboration], Phys. Rev. Lett. 103, 092002 (2009); T. Aaltonen et al. [CDF Collaboration], Phys. Rev. D 82, 112005 (2010).

[4] V. M. Abazov et al. [D0 Collaboration], Phys. Rev. Lett. 103, 092001 (2009).

[5] N. Kidonakis, Phys. Rev. D 74, 114012 (2006).

[6] V. M. Abazov et al. [D0 Collaboration], Nucl. Instrum. Methods Phys. Res. A 620, 490 (2010).

[7] V. M. Abazov et al. [D0 Collaboration], Phys. Rev. D 84, 112001 (2011).

[8] The CDF Collaboration, Conf. Note 10793 (2012).

[9] V. M. Abazov et al. [D0 Collaboration], Phys. Lett. B 705, 313 (2011).

[10] V. M. Abazov et al. [D0 Collaboration], Phys. Rev. D 85, 091104 (2012)

[11] See contribution on top properties at D0 from Christian Schwanenberger, these proceedings. 